\title{
Linx
}

Revue des linguistes de l'université Paris X Nanterre

$74 \mid 2017$

Claudine Normand, une vie dans le langage

\section{Rencontre empêchée à Duino}

Témoignage à partir de bribes

\section{Philippe Depondt}

\section{(2) OpenEdition \\ Journals}

Édition électronique

URL : http://journals.openedition.org/linx/1782

DOI : $10.4000 /$ linx.1782

ISSN : 2118-9692

Éditeur

Presses universitaires de Paris Nanterre

Édition imprimée

Date de publication : 31 juillet 2017

Pagination : 225-239

ISSN : 0246-8743

Référence électronique

Philippe Depondt, «Rencontre empêchée à Duino », Linx [En ligne], 74 | 2017, mis en ligne le 15 avril 2018, consulté le 03 mai 2019. URL : http://journals.openedition.org/linx/1782 ; DOI : 10.4000/ linx. 1782

Ce document a été généré automatiquement le 3 mai 2019.

Département de Sciences du langage, Université Paris Ouest 


\title{
Rencontre empêchée à Duino
}

\author{
Témoignage à partir de bribes
}

\section{Philippe Depondt}

Claudine Normand a laissé des notes, des bribes, qu'elle destinait à un colloque en hommage à Gilbert Lazard, à Duino (juillet 2011) près de Trieste, auquel elle ne s'est finalement pas rendue à cause de sa santé. Sa communication devait avoir pour point de départ la traduction du persan par Lazard des poésies d'Omar Khayyâm. Elle mourut en décembre de la même année sans avoir pu compléter ces notes pour en faire quelque chose de publiable. Il y a une forme de devoir, en plus de la curiosité, à tenter de faire quelque chose de ses derniers écrits, si fragmentaires soient-ils. Je ne suis moi-même ni linguiste, ni historien de la linguistique, mais j'ai vécu à côté d'elle pendant plusieurs décennies; je ne prétends certes pas me réclamer de cette proximité pour reconstituer une «pensée Claudine Normand » authentique! Je ne ferai pas non plus une génétique de sa réflexion : d'autres, dont c'est le métier, le feront peut-être un jour.

2 Que puis-je faire? Nous avons passé le mois d'août 2011 ensemble à Moissac, sa ville natale, dans le Tarn-et-Garonne, et, tous les jours, nous marchions sous les platanes au bord du canal ensemble et discutions : je préparais un cours d'histoire et d'épistémologie de la physique et elle continuait à travailler sur la communication qu'elle n'avait pas pu faire, dans le but d'écrire quelque chose pour le publier. Notre vie était comme cela : la vie affective était accompagnée d'une grande connivence intellectuelle. Elle était bien loin de la physique, mais elle écoutait mes propos avec un mélange de distance et d'intérêt qui rendait ses commentaires extraordinairement stimulants pour moi; j'essayais d'en faire autant pour elle de mon côté.

3 Il s'agit donc de rassembler les bribes: morceaux de texte qu'elle avait ébauchés et souvenirs de ces discussions où j'essayais de l'aider à tenter de construire quelque chose à partir de tout cela. On ne tentera pas reconstituer une pensée qui aurait été perdue car cette pensée n'a pas eu le temps d'aboutir : il est vain de vouloir retrouver quelque chose qui n'a pas eu le temps d'exister, mais il n'est peut-être pas sans intérêt de suivre autant que possible les pistes qu'elle a indiquées, certes de façon parfois énigmatique.

4 Sa façon de travailler incite à la prudence pour qui veut utiliser ses notes pour en faire un objet cohérent; elle pouvait passer des mois sur un sujet donné, à accumuler des notes, 
griffonner des remarques sur des morceaux de papier de formats divers ou des calepins (elle était coutumière du fait lors des voyages en voiture ou en train, par exemple), se trouvant ensuite à la tête d'un paquet de gribouillis disparates, laissant mûrir les choses tout en poursuivant sa quête, glanant des idées au cours de ses lectures et de ses rêveries. Elle se plaignait souvent de la difficulté qu'elle avait "à tout rassembler», "à tout tenir dans la tête en même temps ", il y avait une forme de désespoir dans ces propos et on avait souvent en effet l'impression qu'elle ne s'en sortirait pas. Et, un jour sans crier gare, elle annonçait qu'elle avait écrit la moitié de l'article, que ça s'était en quelque sorte cristallisé, et en quelques jours tout au plus, après un effort de concentration énorme, cela s'achevait, l'article existait sous une forme à peu près achevée. Restait à taper le manuscrit (elle ne tapait jamais directement ses articles), à corriger des détails d'écriture, à compléter les références, ce qui pouvait prendre un peu de temps, mais la phase " d'écriture » au sens de produire un texte cohérent était pour l'essentiel achevée. L'article qui en résultait était en général fort différent de ce qu'aurait pu être un simple agencement des notes prises auparavant, car c'est seulement au moment de l'écriture qu'il était "pensé» dans son intégralité, tout tenu en même temps dans la tête. Pendant l'été 2011, on en est resté à la phase "prise de notes » : fatiguée par la maladie, elle n'a jamais eu la force de réaliser cette cristallisation, l'énergie qu'il lui aurait fallu pour faire l'effort de concentration nécessaire afin de tout tenir ensemble n'était plus disponible. Ce que l'on peut rassembler de ses notes -elle était bien organisée, elles sont à peu près complètes- n'est pas un brouillon d'article : il n'y a jamais eu de brouillon d'article de Claudine Normand, des tentatives avortées d'article, oui, mais des brouillons qui s'affinent au cours du temps, non, pas à ma connaissance. Elle écrivait pour ainsi dire d'un coup et le résultat final aurait sans doute été assez différent des notes qu'elle a laissées.

Il faut savoir aussi que l'écriture manuscrite de Claudine a toujours été assez difficile à lire. Elle-même, lorsqu'elle tapait ses propres manuscrits, devait parfois reconstruire des parties illisibles : à la fin de sa vie, cet aspect des choses ne s'est pas amélioré, loin de là. Claudine avait travaillé sur les manuscrits de Saussure, en particulier les blancs, les phrases dont il manquait des morceaux, un signe de ponctuation entre deux espaces vides, etc. Tentant maintenant de déchiffrer ses propres manuscrits, je me trouve devant, non pas des blancs, mais du super-compact, griffonnages parfois minuscules, raturés, surchargés, avec abréviations mystérieuses, renvois acrobatiques et compléments, corrections, rappels, pense-bêtes dans la marge, dessus, dessous, à côté !

6 Elle était donc partie de la traduction de poésies d'Omar Khayyâm par Gilbert Lazard, puis de Hâfez de Chiraz. Ensuite, Duino lui a évidemment évoqué Rilke et ses Élégies. Dans le même temps, elle lisait les notes de Benveniste qui venaient d'être éditées dans lesquelles il réfléchissait sur la poésie et Baudelaire plus particulièrement. Elle disait aussi que Baudelaire avait sûrement eu accès à la poésie de Khayyâm qui avait déjà été traduit. L'amitié et la correspondance de Benveniste et de Henry Corbin, spécialiste de philosophie islamique, en particulier persane, venait compléter ce faisceau de rencontres possibles... Elle avait lu l'essentiel de Corbin, et s'interrogeait sur le rapport possible entre la complexe angélologie de l'islam chiite et l'épicurisme à la fois mélancolique, gentiment impertinent et alcoolisé de Khayyâm. Quel rapport avec l'Ange de Rilke? Ou les commentaires de Walter Benjamin sur l'Ange de Paul Klee? Ou l'ange de Wim Wenders dans Les ailes du désir auquel elle fait allusion? On comprend alors le défi, le «pari avec elle-même » dit-elle, que de vouloir faire se cristalliser tout cela. Cette cristallisation n'a 
pas eu lieu. Je suis sûr, la connaissant, qu'en bonne santé, elle en aurait tiré quelque chose, comme elle était capable de réfléchir sur une remarque de son charcutier, un panneau routier ${ }^{1}$ ou un graffititi. Elle en était à la fois très loin et à portée de main de cette cristallisation, tant c'était un phénomène brutal quand il avait lieu: un jour elle pataugeait avec désespoir, le lendemain, un seuil était franchi et il y avait un article. En août 2011, le temps de la cristallisation était sans doute venu, mais la force de la réaliser manquait.

7 Quand, lors de nos discussions au bord du canal à Moissac, j'essayais de lui proposer quelque synthèse de ce qu'elle m'expliquait, elle résistait, ça n'allait pas, mais ne proposait pas grand-chose d'autre. Nous ne saurons donc jamais ce qu'aurait pu être l'article prévu, nous n'avons que le témoignage d'une réflexion interrompue. Plus tard, alors que l'espoir de la sauver et qu'elle puisse reprendre son travail s'amenuisait, elle s'exclama un jour avec désespoir : «mais je n'ai pas dit tout ce que j'avais à dire !». En effet. Elle avait encore des choses à dire car, pendant toute sa vie, elle n'a jamais cessé de renouveler ses centres d'intérêt, Saussure, Benveniste, Morris, Culioli, «linguistique douce », ..., et qui sait ce qu'elle aurait pu tirer de la fréquentation de la poésie persane ? Il faut réaliser que depuis la Libération en 1944 - elle avait alors 10 ans -, jusqu'en 2011, année de sa mort, elle n'a jamais cessé de se procurer des livres de poésie et d'en lire, et que, pendant les dernières semaines de sa vie, alors que la maladie ne lui permettait plus de lire par elle-même, elle me faisait lui lire des passages de Baudelaire. S'étant décidée à travailler et à écrire sur la poésie, qu'aurait-elle eu à dire de cette passion d'une vie?

Nous ne pouvons que poser la question et continuer à vivre avec le silence d'une réponse qui ne viendra plus.

Ah, que de siècles sans nous le monde continuera,

Sans nul souvenir de nous ni vestige de nos pas!

Avant notre venue rien ne manquait à l'univers ;

Après notre heure dernière rien non plus ne manquera. (Omar Khayyâm³)

\section{Notes de Claudine Normand}

9 [feuillet non numéroté]

10 Rencontres. Il y en a eu plusieurs -un réseau qui provisoirement a cristallisé sur Duino.

11 J'ai d'abord croisé G. Lazard, rarement (BSL, colloques). Sa gentillesse à Cerisy où essai de faire se rencontrer et se connaître un peu mieux la linguistique gén. contemp. et comparative. B. rassembleur? Mon ignorance du persan et mon indifférence à la typologie.

121 pure rencontre : les quatrains de Khayyam présentés et traduits par G. L[azard] = 1ère vraie rencontre avec la poésie persane et idée que celui qui traduit un poète est sans doute philologue mais accessibilité littér. part. qui n'est pas un ingrédient si courant chez les grammairiens.

13 À l'occasion d'une autre rencontre, banalement universitaire, je parle à G.L. de ma découverte et il m'envoie Hafez. Allusion sans plus au souffisme, mystique chiite qui rappelle la façon dont j'ai croisé Corbin et l'angélologie $\rightarrow$ envie d'en savoir plus et plongée (trop rapide, juste le désir d'en savoir plus) sur cette histoire d'anges (sans dépasser l'étonnement naïf sur l'angélologie) évoqués si souvent mais restés connaissance superficielle, sans questions véritables. 
Sur ce une sorte d'appel, un rappel plutôt d'une lecture, Duino, d'autrefois, superficielle et rapide, tout ce qu'une préparation de concours me permettait à cette époque : Les Élégies, Rilke, ce noceur[?] qui se renverse si facilement en lyrique. Je retrouve dans une strate ancienne de ma bibliothèque une édition (Émile Paul) intitulée Poésie ayant conservé un modeste $\times$ (au crayon) ma signature de l'époque (1959) qui [?] cette 1ère lecture.

Réveil soudain de moments bien oubliés.

Sur le moment (et encore auj. ) presque énigmatique des élégies et des sonnets à Orphée (en exergue « chanter c'est être »)

Ont suivi + abordables la mélancolie romantique du cornette Christoph Rilke, les lettres à un jeune poète (travailler ! être soi-même ! ça, me semblait-il, je savais) et surtout, assez vite abandonné Les Carnets...

Ici le réseau des rencontres devient labyrinthique

Gilles $^{4}$

Wenders $^{5}$

relire attentivement

les anges - et l'ange de la 1ère élégie $\left(4^{\circ}\right.$ vers de la $1^{\circ}$.

[verso]

peu de rapport avec la tradition bachique mais qque ombre portée du soufisme et de l'iman caché?

Je reviens à Corbin, ami de B. (lettres-photo) en lisant trop rapidement son hist. de la pie islamiste je comprends un peu mieux les longues lettres (perdues?) que m'envoyait cet étudiant étrange ${ }^{6}$, qui lors d'une soutenance se disputa violemment avec Ali Ben Maklouf, spécialiste d'Averroës. Ne pas confondre sous pretexte d'anges le rationalisme et le mystique, Averroës et Avicenne? Que de connaissances absentes et qui restent si incertaines! Des anges je n'en vois pas chez Omar ni Hafez, mais est-ce prudence?

Mais voilà que je retrouve les anges dans la production inquiète du début du XXème s., référence svt explicite à Baudelaire encore plus qu'au romantisme allemand

7 Et chez celui-ci chez qui on l'attendrait le moins, W. Benjamin, un temps lié (mais gd ami de Scholem) à la très sérieuse école de Francfort en exil mais aussi W. Scholem et la mystique juive. Il se [?] que c'est une lecture d'insp. messianique, sa correspondance avec W. Scholem, que j'apprends à quel point B. tenait à cet «ange du bizarre » qu'il avait découvert est-il dit cf Poe et surtout dans le tableau de Paul Klee. Était-ce le même que celui des Élégies, + rassurant ou plus inquiétant ? [retrouver le texte de B. Brun]

B (retour à Baudelaire, tableaux parisiens, Rilke, les cahiers, l'exil et le nomadisme des élites de la Mittel Europa d'une guerre à l'autre, Zweig et son suicide pendant de celui de Benj., la mort précoce de Rilke [?]

9 Derrière (à peine) tout cela la politique, l'histoire et ses victimes, et nous retrouvons alors deux qui ont (par miracle? astuce ? obstination?) échappé au sort des victimes et ont pu reprendre et aboutir travaux qui nous réunissent ici : Benv. exilé en Suisse, G. Lazard déporté.

Or je les ferai se rencontrer une nouvelle fois ici, non que G.L. ait ignoré B., évidemment, mais ce qu'il ne pouvait connaître jusqu'à il y a peu (et encore) c'est la préoccupation intense de B. sur la poésie. 
Se seraient-ils rencontrés sur ce point et sur Baudelaire? On peut toujours rêver ces rencontres... Je les ai mélées aux miennes, évidemment personnelles et idiosyncrasiques, tjrs embrumées d'ignorance et illuminées [?] de désir de savoir et de comprendre.

« traversée ça et là par de brillants soleils »

[feuillet non numéroté]

Cette $\mathrm{K}^{\mathrm{ion}}$ est le résultat d'un pari avec moi-même évidemment, le pari d'une rencontre avec un Gilbert Lazard que je ne connaissais guère que de loin. Je m'explique : d'abord sur les propriétés sémantiques que j'attribue à ce mot rencontre : il suppose pour moi hasard et imprévu (en qque sorte l'inverse même d'un colloque annoncé, d'un programme sérieusement fixé, voire minuté). Celui qu'on a rencontré n'était pas annoncé ni attendu et bien différent du 1er venu qu'on croise dans la rue ou même de ceux qu'on connaît ou retrouve sans surprise dans une assemblée, il retient l'attention par quelque trait original qui le différentie de ceux qu'on connaissait déjà ; « original » et « différence » ces termes supposent qu'ici qqu parle de qqe chose qui le concerne personellement. En ce qui me concerne ce qui a tt déclenché, me donnant le désir de participer à ce colloque et d'en faire une rencontre, c'est le nom même de Duino. Il m'est revenu d'un passé déjà lointain, de ces années 50(?) où j'avais trouvé sur les quais « les Elégies de Duino », poèmes d'une écriture mystérieuse presque hautaine dont l'original allemand m'était barré. Je ne connaissais alors ni Rainer Maria Rilke ni cette ville italienne au nom fluide. Je n'ai pas cherché d'autres informations et ai lu sans les comprendre d'abord ces Elégies et les sonnets qui me parlaient d'anges secourables ou effrayants, ces sonnets [?] d'orange se faisaient chair et joie ([?] l'orange...)

[verso]

- l'Orient (le voyage, la dernière étape) cf soufi orient

-angélologie - Corbin

-Kayyam : l'ivresse

la vie est un voyage trop bref dont il faut chasser l'angoisse

l'art: le potier

la beauté des formes

la matière et la continuité vivante

revendication de liberté (Ronsard) de créer et de vivre

[feuillet numéroté 1]

\section{Rencontres}

Annoncer une rencontre c'est laisser ouverte la place du hasard et de la surprise; du risque : aussi désagrément d'ennuyer par des réactions trop personnelles, on ne peut attendre des rencontres qu'elles soient toutes heureuses ou surprenantes, certaines sont dites mauvaises ; d'autres celles qu'on a retenues généralement, ont pu être éblouissantes mais sans suite. «Ce fut comme une apparition» dit Flaubert de Mme Arnout que F. découvre sur le pont du bateau de Rouen et presqu'aussitôt leurs regards se rencontrèrent; on sait que la suite ne fut pas à la hauteur de ce $1^{\circ}$ mouvement. Elles peuvent aussi, bien que permises, contrariées par les circonstances, et ne laisser que des traces rêvées. Ce fut le cas [?] à Duino en juillet 2011, si bien qu'il ne s'agira dans ce petit exposé que de celles que j'ai retrouvées ou réveillées à l'occasion de cet hommage et dont j'aurais aimé parler. Promettre des rencontres est bien risqué car il pourrait ne s'agir ici 
que de celles personnelles que j'ai faites ou réveillées à l'occasion de ce petit exposé de circonstance (où peut-être ne seront évoquées que mes propres surprises).

Précisons d'abord le thème enchevêtré de ce discours. Nous sommes [?] ici y rendre hommage à G. Lazard, chacun ayant ses raisons personnelles pour ce faire. En ce qui me concerne, on pouvait s'en étonner car j'ai fait sa connaissance tardivement, étant depuis longtemps déjà engagée dans des analyses de ling. gén. très tôt limités à Saussure, Benveniste et plus récemment Culioli, c'est à dire loin du comparatisme et de la philologie indo-iranienne [?], loin aussi des mé $\theta$ concepts plus récents de la linguistique générale qui l'occupent. C'est à l'occasion d'un colloque Benv. à Cerisy que j'ai rencontré G. Lazard, puis une deuxième fois à Cerisy encore où nous avons eu l'occasion d'évoquer des quatrains d'Omar Khayam qu'il a traduits et présentés dans une jolie petite collection de poésie appelée Orphée (et que je connaissais) (ed. La Différence). J'en reçus un exemplaire de sa part ainsi que, dans le même domaine et la même inspiration "Cent un ghazals amoureux » de Hâfez de Chiraz (Gallimard). Quand je reçus la proposition de participer à cet hommage, je me trouvai ravie mais d'abord un peu embarrassée n'ayant aucune compétence pour aborder le domaine iranien, et guère pour proposer quelque nouveau questionnement de ling. gén. susceptible de s'accorder à ses travaux actuels. Mais la poésie nous rapprochait, d'autant que s'opère ici la $2^{\circ}$ et pour moi la + surprenante rencontre, une sorte d'appel venu du nom même de Duino: [?] par une $1^{\circ}$ rencontre mais le souvenir ébloui et resté confus des Élégies de Duino suivies des sonnets à Orphée souvenir très ancien de ma découverte de Rilke dont sur le moment (je parle des années 50) je n'avais pu rien faire d'autre que d'en garder vive l'émotion : «Qui donc, si je criais, parmi les cohortes des anges / m'entendrait?» [?]on m'invite à Duino cri de solitude et appel de la $1^{\circ}$ Élégie répétée dès la $2^{\circ}$ Élégie : "Tout ange est terrible ». Que pouvaient être ces anges, cette présence à la fois funèbre et pleine de promesse inquiétante : « Afin qu'un jour, au bout de l'entendement terrible, je puisse chanter l'allégresse et la gloire sous l'approbation des anges » ( $10^{\circ}$ Élégie p. 37 ) [ici, ignorance de l'islam et angélologie. Corbin, Benv., mais des échos cf les poètes Baudel. L'ange plein de gaieté, Gounod....]

À première vue peu de rapport ici avec la poésie persane, mais le rapprochement inattendu que m'offrait le hasard de mes pensées et de mes souvenirs m'oriente [?] vers un goût commun pour la poèsie et le monde intermédiaire qu'elle veut dire, préoccupation qui n'est pas absolument banale dans le travail des linguistes. Ici s'amorce un réseau tt personnel subjectif à partir de quelques repères peut[?]-être partageables (des liens dirait-on auj. à partir de mots-clés) et d'abord le nomadisme de Rilke, qui sans connaître la pauvreté absolue a toujours dépendu d'amis protecteurs généreux, de femmes surtout, ainsi, à Duino, la princesse de Thun et Taxis. C'est ainsi qu'une [?] de R. présente l'étape de Duino (Mitsou p.7) qu'après une errance presque fébrlie, retrouvera pour y terminer les Élégies en 1922. On sait que Kayyam comme les troubadours de tous les temps a dépendu lui aussi de faveurs, tjs susceptibles de manque, disparaître et exigeant parfois quelque prudence. Un point commun donc, dans des conditions certes très difftes, mais qui nourrit, chez l'un et chez l'autre, le symbolique du voyage : chercher le nouveau, l'inconnu et dans l'ivresse, le mouvement et le lieu où pourra se produire l'éblouissement rêvé. C'est d'avance un thème romantique encore présent chez Baudelaire pour (Palmyre) métaphoriser la mort « Plonger au fond du gouffre... trouver du nouveau », ( $\mathrm{O}$ mon vieux capitaine... nos cœurs que tu connais sont remplis de rayons ») 

fine » disait Baud. retrouvant ici peut-être Kayyam tel que ses amis romantiques l'ont découvert dans une traduction dont Th. Gautier fit un c.r. enthousiaste. G. Lazard fait du vin chez Kayyam ainsi que chez Hafez de Chiraz un thème répétitif, qui n'appelle pas seulement l'oubli dans la taverne mais évoque l'extase dont rêve le poète. (cit. p.151 Hafez) emblématique de l'expérience extatique attendue dans la création artistique ... n'a garde d'aspirer à se désenivrer. On reconnaît B. « enivrez-vous... » tt autt que Kayyam (p. 25, 29), avec chez ce dernier le rappel obsédant de la [?] de la vie, vieillesse et de la soudaineté de la mort [?] (sur ce pt cf le long roman historique d'Amin Maalouf: Samarcande) que [?] croyait bien connaître depuis la tradition bachique et [?] Ronsard mais au lieu de mélancolie rêveuse de ce dernier « regrettant mon amour et votre fin dédain » qui évite les évocations trop pénibles [?] l'insistance sur l'ivresse, cf Kayyam et cf Hafez, est surtout l'occasion d'affirmer liberté de pensée et refus des contrainte rituelles de tte religion : impertinence jusqu'au blasphème. Lit-on encore auj. Kayyam à Téhéran? (cit)...

50 [feuillet 2]

51 le « déréglement de tous les sens » annoncé et [?] rassemble ainsi pour moi dans la même exaltation, rêvée ( plutôt que réalisée sans doute des poètes également révoltés par l'ordre moral qu'impose une religion corsetée par le rituel. Kayyam n'hésite pas à la refuser à ses risques et périls et on a pu lui reprocher cert. quatrains blasphématoires ; (cit) Baudelaire lui dut l'affronter dans tte sa trivialité (cf l'acte d'accusation des Fleurs du Mal par le procureur Picard). Si Rilke, à la différence parfois de Baudelaire, ne semble pas préoccupé de régler ses comptes à de [?] religieuse contraignante et hypocrite qui n'est évoquée que de façon vague ; ce qui est + que suggéré, promis dans le Élégies et dans les Sonnets c'est la promesse messianique d'une révélation par l'art, chemin possible de l'extase mystique (cf B. [?] extase, ange). Les commentateurs de R. (cf Paul de Mace) ont élaboré (cit) sur ce thème: virtuosité [?] de la figure ou expérience mystique? On pourrait se poser les mêmes questions que Baudelaire (cit les phares) ou la méta $\varphi$ du souffre (cit). Chez O.K. et Hafez -moins lyriques que les deux romantiques dont je les rapproche peut-être abusivement, on a pu aussi s'interroger sur l'attrait exercé par la mystique des soufistes (cit Grolleau). Mais si chez Rilke et B. l'éblouissement espéré semble lié à l'Ange qui accomplira la promesse (ce même « ange écarlate du matin » dont l'appel (dans le Temps retrouvé) (cit p. 371) assure Proust de la force de son œuvre, jusqu'ici, je n'ai pas trouvé chez les deux poétes persans l'affirmation explicite de sa présence sur le chemin de l'illumination. Pas de soufisme chez Kayyam assure Ch. Grolleau ; et G. Lazard de Hafez : « est-il ... et il voudra ». Mais chez les deux et sans doute d'autres, baignés de la même tradition soufie, un sentiment joyeux du concret, qui loin de [?] à écarter la mort permet de vivre dans la plénitude de la vie, précisément parce qu'elles sont saisies dans une continuité concrète. 
$62 \leftrightarrow \mathrm{O} . \mathrm{K}$.

\section{l'émotion (sensorielle)}

Chez Rilke [?] messianisme des Élégies et des sonnets à Orphée a suscité le débat cf les commentateurs : virtuosité rhétorique de la figure ou expérience mystique (cf Paul de Mace, intro) dont l'appel serair sous-jacent sous couvert d'ivresse à la poésie persane, refusant le dogmatisme religieux en lui opposant la liberté du poéte (cit.)

[retrouver chez R. et $\mathrm{B}$. passage où ange et mort semblent accomplir la promesse et chez O. sous couvert d'ivresse K. ?]

[?] de célébrer les objets du monde $9^{\circ}$ Élégie p. 370-371 la [?] familière l'existence sur[?]

3 l'association constante de la mort (présence des morts) dans la jouissance des objets de la vie. Le [?] de Rilke. Cf image constante cf $\mathrm{K}$

$64 \downarrow$ O. K. ne s'impose pas de regarder l'horreur de la charogne B. $\rightarrow$ sublimée dans l'art (la forme) (cité en [?] 2 fois par R.) : exorciser l'angoisse par ce « double empire » il ne s'agit ni de fuir ni d'oublier (cf. la dentelle et la cruche).

5 Il ne s'agit plus de méta $\varphi$ mais du concret lui-même dans sa quotidienneté. Celui qui semble fuir l'ange de l'histoire de Benjamin. Celui qu'un ange ne peut choisir de la fiction de Wim Wenders.

66 [Cette épaisseur de chair que Benv. voulait arriver à dire mouvement de vie, et en témoignage ses ms enfin retrouvés sur le « pbe poétique »]

[feuillet 3]

(trop pénible), Kayyam et plus tard Baudelaire et Rilke introduit la mort dans le concret de la vie, mêlant au plaisir du vin et de l'amour, la présence transformée des morts : (le thème de) l'argile dont est faite la cruche, rattache relie le poête aux morts dont se nourrit la terre.

Ce thème de la continuité concrète des morts aux vivants qui évite de souligner la brutalité irrémédiable du changement, introduit dans ces quatrains (plus que quelque leçon de morale) une sorte de douceur un épicurisme raisonnable en même tps qu'une invitation appaisée à profiter des plaisirs non en [?] mais en [?] (O. K. p. 57,59-61)

$70 \mathrm{r}^{*}$ On retrouverait difficilement ce thème cf. B. le révolté même si, devant le [?] qu'il fait répugnant d'une "charogne», termine sur cette affirmation de la victoire de l'art (dernier vers)

71 Mais, chez Rilke, curieusement, je crois pouvoir retrouver cette association de la mort et $\mathrm{d}$ ela jouissance de la vie concrète, jusque dans l'image famil. à K. du potier pétrissant l'argile (370 371)

[feuillet non numéroté]

(fil conducteur?)

-partir de Rilke et Khayam. Éloignement extrême ou arrière-plan commun qu'on peut imaginer (ou reconstruire cf Amin Maalouf) : le nomadisme [?] et l'angoisse/la présence de la mort. Sous l'îvresse chantée et proclamée contre tous les dévots inquisiteurs du persan, sous le travail solitaire et obstiné, svt désespéré du romantique pris dans un monde d'où disparaît la spiritualité

(ici citer K. : interrogation sur le soufisme

commentaire de Th. Gautier. Le romatisme 
le soufisme sous-jacent ou explicite cf Hafez traduit par G. L., cf exergue, cf lettre de Corbin à B. et [?] de ces débuts de l'iranologie)

-sur Kayyam et le soufisme cf Corbin (intro)

-Rilke n'a pas l'appui d'une tradition de sa quête spirtuelle qui rencontre, mais de la solitude romantique -les [?] $\varphi$ - religieuses, et suggèrent alors (pour donner un ordre aux phénomènes mystérieux: la présence soudaine de "fantômes » ( $\mathrm{cf} A[?]$ ?) les termes consacrés de la religion monothéiste sous le [?] de l'ange cf $1^{\circ}$ élégie. À la foi [?], révélation, appel, frayeur, dépassement.

-moins explicite cf Baud., moins [?] mais présence constante: solitude -angoisse de comprendre- attente de la révélation $\leftrightarrow$ la mort (le voyage) $\downarrow$

-rapports étroits Rilke-Baud. (rappel la dédicace de B. à Th. Gautier $\leftrightarrow$ romantique cf [?] la boucle d'Ombre)

cf tableaux parisiens [?] des Carnets ( [?] [?] d'un désir sans trêve)

Mais quel rapport de tout ceci avec Benv., qui [?][?] ce qui a suscité ma rencontre avec G. Lazard

5 -le persan d'abord, mais sur les relation de L. et Benv., autour ou sur le persan, je ne sais rien, n'ayant pas interrogé L. $\leftrightarrow$ ma hardiesse imprudente. Difficile d'imaginer qu'ils se soient ignorés (cf Tous 1980). Je passe ici par l'intermédiaire de ce que je connais de Corbin (peu), ami de Benv. (cf lettres) et dévot de l'iranologie encore au début des années 50, avant que la pensée de B. ne soit recouverte par le souci « moderne » de la ling. gén. et d'une $\theta i e \rightarrow$ on n'a gardé que ça. Mais Benv. (à qui Corbin rappelle leur amour commun de la poésie persane) n'avait pas oublié, même embarqué dans la [?] des structures, que la poésie[?][?] d'une quête spirituelle jamais abandonnée et que continue à être exigé une recherche spécifique sur ce qui soutient cette tradition du " [?] poétique ». C'est ce qu'il suggère (affirme !) au [?][?][?] du Sme en 68. Ce qu'il n'a pas eu le temps de faire -mais [?] bcp de traces.

[verso]

travail actuel sur Baudel. Benv. à partir des ms, et des questions (svt obscures) de Meshonnic, dévôt de Baud et de Benv. et représentant solitaire d'une autre branche de cette tradition herméneutique passionément attachée au texte, trad. de la Bible

cf lettre Corbin $\rightarrow$ B. sur Kayyam

[feuillet non numéroté]

B ms

-devient chantre des sensations et de l'émotion

le vice n'est pas traîté à la manière de $K$. plutôt suscité par désespoir/misère, aucune gloire mais aussi extase, îvresse mystique de l'amour et de la création art.

Chez les 3 même qd pas nommée, présence de la drogue

l'îvresse peut recuvrir plusieurs modes, paradis artificiels ("le vin de l'éternité » de Hafez) et même l'nethousiasme mystique.

Hafez ambiguïté [?] ironique (207)

-boire [?] qu'on ne comprend rien (210) et qu'il n'y a rien d'autre à attendre (213) 
-l'enthousiasme p. 218

cf Rilke pas l'îvresse pour l'ennui ou la tristesse, la misère du nomade exilé. n'attend une ouverture sinon une issue que de l'art et du travail qu'il exige cf Rodin mais peut évoquer la splendeur (id. B. [?] perdus... cf si Benv. Cite)

-Rilke retrouve B (explicitement ou presque)

[?][?] dans atmosphère déprimante et fascinante de Paris : misère , maladie, mort cf début des carnets

les rencontres [?] (cf le visage arraché fantasme hallucination B. les veuves rêves regrets)

-Rilke ne retrouve pas Kayam portant connu des romantiques cf Th. Gautier ([?][?])

ni Ronsard, la tradition anacréonique [?] romantique inquiètante étrangeté (carnets)

la mort est là tt le temps $\neq$ ce rappel assaisone le plaisir,à la manière des libertins blasés (cf Bauer du sang de la volupté de la mort) stimulés par la transgression, le péché

cf K. c'est libre est joyeux

cf R. c'est peu présent mais pénétre le concret, l'enrichit

(opposer l'argile du pot / la dentelle ) | en fait partie, le double

$\uparrow$ travail $\mid \downarrow$

| cette théorie du « double empire »

pas d'anges chez K., ni H. alors que présence | l'ange de Élégies

essentielle de l'islam | terrible présence

dans B. ? cf anges pleins de gravité[?]...

[feuillet non numéroté, plié en deux : 4 pages \#1 sans ordre clair]

Comme son titre l'annonce cette intervention se situe sous le signe des rencontres et ne se justifie que par elles.

N'étant ni philologue, ni comparatiste, non plus que spécialiste de quelque lgue que ce soit, je ne dois le plaisir (et l'honneur) de me retrouver ici qu'à une série de rencontres dont une décisive, qui m'a en quelque sorte invitée à suivre l'appel de son nom : Duino

Ici se mêlent souvenirs déjà bien anciens.

[\#2]

9 Y-a-t-il eu rencontre fugace, ébauchée, comme un appel à continuer sur un autre chemin autrefois soupçonné ? Retrouver plutôt et tâcher de comprendre ce qui serait l'essentiel ? Mais ne pas oublier un des deux anges de Wim Wenders, l'un choisit l'amour terrestre, renonçant à entendre ce qui reste caché aux humains ordinaires. Il choisit une artiste exposée à ts les dangers, une trapéziste , entre ciel et terre dans le monde intermédiaire.

[\#3]

Exorciser la mort, l'angoisse de cette pensée par l'oubli provisoire de l'îvresse et de l'amour, du plaisir amoureux et (allusivement?) par l'attente d'une illumination, par l'extase mystique que devra apporter au moins la mort

B. les sonnets sur la mort, élévation, etc....

et surtout le voyage

le thème du voyage (retour vers l'Orient) chez les soufis sinon explicite chez $\mathrm{K}$. 
[\#4]

Rencontres lumière. p. ds

$\theta$ communs

-voyages

R.la création difficile , la voie du travail (cf Rodin, Proust) mais aussi la mort apprivoisée dans le concret vivant, les objets, les fleurs, les fruits([?] la cruche et la dentelle, cf Rimbaud fugitivement). La mort nourrit la vie, le double empire, le monde intermédiaire. Celui de la mystique soufie.

(B. cet après-midi qui n'a jamais de fin)

Et Benv. ? Corbin à l'abri, mais retrouve B. et cherche à l'entraîner. La poésie, s'est autre chose, ce n'est pas de la sémiotique, positive, des règles, des classements.

La sémantique? le sens? mais comment rendre compte de ce passage mystérieux et nécessaire par les formes sémiotiques? $\neq$ simple traduction, transposition. Quoi ? Ce travail de la formecf Baudelaire, mais chez B. il n'y a pas d'ange vers lequel crier. Reste à retrouver la grâce de la Parole, chaque fois unique, création d'un monde, du Monde. (mais surtout par la communication) La mystique juive de Scholem? L'ange de P. Klee qui a fasciné Benjamin. Le monde bouleversé de l'entre 2 guerres et après guerre, à la recherche d'une éthique et d'une esthétique. Ouvert à tous les messianisme

Lazard $\leftrightarrow$ Benv.

Kayyam - Hafez

Duino (1912-1922) (cf préface à Mitsou)

Quoi de + éloigné K. R. ?

mais de l'un à l'autre : une attente : la création. la poésie.

Le choix d'un monde concret, à la fois immédiat (le vin, les fruits, l'amour) et ombre et

Entre les deux le romantisme allemend et ce qui marque la fin B. Présence de B. chez R. en

les cahiers. au titre du concret [?] le + dur, mais aussi la solitude, la tristesse de la grande ville, la mort partout (sonnets)

le travail de la forme, seul accès à création (cf Rodin)

Kayam [?] par les romantiques. ni B., ni R. n'en parlent et pourtant il y a des aspirations communes sous un [?] $\neq$ : liberté (dans les drogues)de la [?]

de l'ordre moral $\rightarrow$ blasphèmes ou impertience

Sous et malgré la tristesse, la solitude, l'ouverture vers la lumière de l'inconnu qu'apportent l'am et la mort (Venez vous enivrer...)

-la place de la mort

-K. ₹ funèbre même si la pensée (rappel) de lamort est très présent

\# simple ivrogne malgré ce qu'il dit de lui-même et de ses compagnons d'îvresse

₹ Ronsard et la tradition épicurienne. En profiter tant que c'est possible $\rightarrow$

[feuillet non numéroté]

-nomadisme $\leftrightarrow$ dépendance et incertitude 
$\downarrow$ Zweig

-O. Kayyam

vin

$\uparrow$

-voyages réels chez R. tradition évoque [?]

-voyages fantasmés chez B. $\leftrightarrow$ romantisme et nécessité Benjamin

dans le Voyage/la Mort qui vient à [?] de la pauvreté

fuir l'angoisse. (re)trouver le lien de la création possible. le refuge (cf début Mitsou)

-dans le vin - l'îvresse $\rightarrow$ extase?

Il ne s'agit pas d'ivrognes mais de poètes qui cherchent à trouver (retrouver) une joie déjà vécue, la fixer (cf mais comment? sonnet) l'éterniser

enchaînement

liberté (de pensée) Laz. p. 105

provocation contre contraintes d'ordre religieux

$\rightarrow$ liberté d'esprit et de corps $\rightarrow$ création blasphème (+ impertinence que violences G.L. p.91/Grolleau p.95 , [?] + qu'angoisse G.L. p. 107, 111, 113, 115)

(procès de Baudelaire) [?] de l'impiété,

d'obscénité, de mauvais goût

voie : une charogne R. citant 2 fois B. = [?] de la rupture du moderne (cf Cézanne Rodin)

le concret les objets, les corps jusqu'au + pénible

la mort présente ds la vie

-O.K. ne s'en lamente pas au contraire (G.L. 32-41)

-les morts sont présents dans la cruche du potier et de [?]

$\leftrightarrow$ imaginaire. funèbre ? Grolleau p. 116

jouir pleinement de la vie qui passe si vite, de tous les

plaisirs $\neq$ tradition bachique, plutôt Épicure

$\neq$ Ronsard - après il ne sera + temps $\rightarrow$ regret et tristesse

mais le plaisir zctuel est comme relevé d'être mêmé au souvenir des morts qui restent présents dans le concret de l'argile travaillée, du vin parfumé, des fruits nourris de la terre (citer l'orange) $\rightarrow$ un monde intermédiaire, le double empire. Sur ce thème Rilke rejoint et développe les persans ( $\mathrm{cf} \mathrm{Hafez....)} \rightarrow$ la cruche et la dentelle

7 ( une dévotion créatrice au concret et au travail qui a fait [?] (cf Proust)

-dans le monde ouvert à toutes les sensations, créer les formes qui le retiennent, les retrouvent, leur donne une éternité, mis accepte l'incertitude, le labeur, les déceptions dont Kayam e parle jamais comme si ses vers coulaient aussi faliles que le vin

(les romantiques sont passés par là)

écouter la crainte de la mort et l'angoisse : par l'apaisement vouloir ce qu'on a, rien d'autre. repos? extase peut-être parfois ? poésie? (p. 119 la dernière chez G. Laz.)

[feuillet non numéroté]

-Kayyam-Rilke 
-le concret, l'expérience, en jouir avant de mourir mais $\neq$ chansons d'ivrogne (cf. Gautier)

il s'agit chez les deux de museler/maîtriser l'angoisse chez K. ironie, légèreté, chez R. une quête longue, obstinée qui des Élégies et sonnets semble abboutir à l'apaisement dans le fantasme du « double Empire » : la mort est déjà là dan sla vie, mais $\neq$ angoisse -une sorte d'entre-deux, de double connaissance avant l'heure (le monde imagine[?])

$\neq$ la tradition anacréonique, ni Ronsard.

Le temps passe

On ne l'arrête pas. de chanter en qque sorte un [?] pressenti d'une suite, déjà présente -la mort est devenue familière. L'argile est faite des corps des morts, cendres, oui, mais par là elle reste vivante, pque elle garde présente la vie. Le concret est plus riche que le toucher passager. Il y a ce Afez plutôt + les femmes

l'amour caché

-les 2 Өèmes chez Baudelaire mais n'y tiennent pas la même pplace

chez B.: l'art est le véritable objectif par quoi la vie prend sens. par le travail (l'expérience) id. chez Rilke (romantisme)

\section{NOTES}

1. Il y a bien longtemps, il y avait sur l'autoroute A6, à quelques kilomètres de distance, deux panneaux : le premier indiquait "Vézelay, colline éternelle" et le second "Buse, rapace utile". La linguiste, qui était aussi une femme pleine d'humour, s'amusait infiniment de cette séquence étrange.

2. En 2010-2011, elle a beaucoup médité sur un graffiti qui disait :

FUCK

l'amour et le quotidien

le premier mot en lettres-bâton, le reste en cursives très « école primaire ». Elle rapprochait cela du vers de Maïakowski qui disait en substance : «le canot de l'amour s'est fracassé contre le rocher du quotidien " (elle se posait aussi la question de la traduction du russe, ce canot lui paraissait étrange). Elle me demandait tous les usages du mot « fuck» en anglais et s'interrogeait sur les raisons de l'emploi de ce mot à la place d'un éventuel équivalent français. Ce graffiti avait aussi la particularité d'avoir été écrit par terre sur une passerelle que tout le monde piétinait en passant : elle se demandait s'il y a avait une intention là. Elle est allée jusqu'à laisser un petit mot de demande d'explication accroché à côté : il n'y eut jamais de réponse.

3. Omar Khayyâm, Cent un quatrains, traduction G. Lazard, Orphée (1997), n 44 : un marque-page était resté à cette page.

4. Il s'agit d'un des fils de C. N., amateur de Rilke.

5. Les anges dans Les ailes du désir.

6. C'était à la fin des années 1980 ou au début des années 1990, le mémoire de maîtrise d'un étudiant passionné d'Henry Corbin et de philosophie islamique. Il envoyait à C. N. de longues lettres enflammées qu'elle lisait avec beaucoup d'attention sans vraiment comprendre ce qu'il cherchait. Pour la soutenance, elle avait fait appel à A. Ben Maklouf pour l'aider. Elle en était 
revenue assez troublée. L'étudiant en question a continué à lui écrire pendant quelques temps puis, à ma connaissance, n'a plus donné signe de vie.

\section{AUTEUR}

\section{PHILIPPE DEPONDT}

Sorbonne Université, UPMC Univ. Paris 6, UMR 7588 INSP 